\title{
Molecular Magnetism with Fullerene Building Blocks: the race is on for High $T_{c} s$
}

\section{Mihailovic}

Josef Stefan Institute, Jamova 39, Ljubljana, Slovenia

Ferromagnetism in organic materials with only $p$ electrons is a relatively new phenomenon having been discovered only a few years ago. Recent discoveries of fullerene charge-transfer compounds with Curie temperatures beyond the exotic experimental domain of dilution refrigerators, together with progress in our understanding of the magnetically ordered state, opens the field to discoveries of potentially useful organic ferromagnets.

\section{Introduction}

Macroscopic manifestations of collective quantum states like superconductivity and ferromagnetism are often the most interesting phenomena in solid state physics. However, they are also the most difficult to understand. In the case of ferromagnetism, the magnetic properties of magnetite have been known to humans for millennia, but an understanding of the phenomenon had to wait until the advent of quantum mechanics and the ideas on exchange coupling of Heisenberg, Dirac, Neel and VanVleck.

Nature is full of surprises and the existence of ferromagnetism in organic materials - an area where there has been significant experimental progress in the past few years - is definitely one of them. The purpose of this paper is to review the recent work in one of the most interesting areas in the physics of magnetic phenomena this decade, namely the ferromagnetism in fullerene compounds first discovered in 1991 by Fred Wudl and collaborators at the University of California in Santa Barbara [3].Theirdiscovery of ferromagnetism with a Curie temperature of $16 \mathrm{~K}$ in tetrakis-dimethylamino-ethylene- $\mathrm{C}_{60}\left(\mathrm{TDAE}^{-\mathrm{C}_{60}}\right)$ was an order of magnitude higher than the previous record and brought the research field from the realms of the esoteric into the mainstream.

M. Mihailovic is associate professor of the University of Ljubljana and senior research fellow of the J.S. Institute. He leads two research groups working on high TC superconductivity and fullerenes. He took his D.Phil. at Oxford on the Jahn-Teller effect in oxides and Raman studies.
The structure contains unusually short distances between adjacent $\mathrm{C}_{60}$ buckyballs of 9.99 [6] which is believed to lead to coupling between spins localized on $\mathrm{C}_{60}^{-}$ions and the formation of a ferromagnetically correlated spin state.

Since TDAE- $\mathrm{C}_{60}$ was discovered, there has been significant experimental progress, particularly in elucidating the magnetic and electronic ground state properties of this material. However, very few new compounds have been found to show similar behaviour, and until very recently TDAE- $\mathrm{C}_{60}$ remained the compound with the highest $T_{c}$. For example TDAE- $\mathrm{C}_{70}$ and many different TDAEdoped $\mathrm{C}_{60}$ derivatives show no evidence for a ferromagnetic (FM) state down to $4 \mathrm{~K}$ - which is also true when TDAE is substituted with other electron donors [7] - and although there have been some reports of possible ferromagnetism at higher temperatures[8], up until quite recently such unidentified ferromagnetic organic compounds (UFOs) have so far failed the reproducibility test. The current record thus stands at $20 \mathrm{~K}$, which was set very recently at our laboratory and is for cobaltocene-3-aminophenyl-methano[6o]fullerene [9], whose properties are currently being intensively investigated.

\section{Unusual magnetic properties}

When the magnetic properties of the material were first measured at low temperatures [3], the researchers - who were perhaps hoping more for a diamagnetic signal indicative of superconductivity - found a rather unusual cusp in the susceptibility with an onset at $\mathrm{T}_{c}=16 \mathrm{~K}$, clearly indicating that below this temperature some kind of collective spin ordering was taking place. (Such a curve is shown in Fig. 2 for the case of a single (Fig. 1).

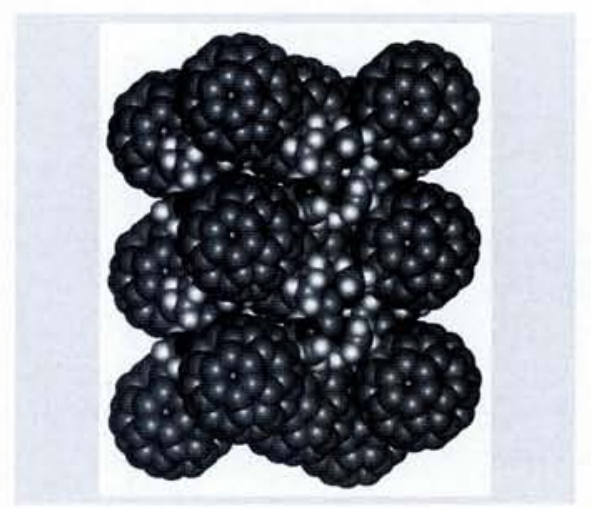

Fig.1. The crystal structure of the molecular ferromagnet TDAE $C_{60}$

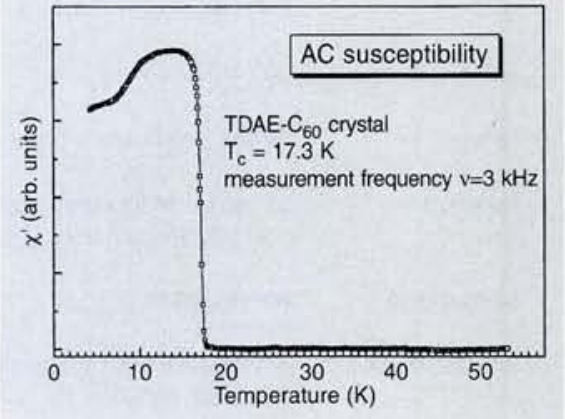

Fig. 2. The magnetic susceptibility of a single crystal of TDAE- $C_{60}$ in the absence of external field showing the onset of a spin-ordered state below $16 \mathrm{~K}$ 
crystal.) Moreover, the magnetization of the sample showed a non-linear curve as a function of magnetic field such as is found in soft ferromagnetic materials. (In these, there is little or no remnant magnetization in the absence of external field, a property which is useful for making transformer cores, for example.) These properties, together with an observed increase of $\mathrm{T}_{\mathrm{c}}$ with magnetic field - another property typical of ferromagnets, led them to propose that the discovered material is a ferromagnet. Subsequent measurements have confirmed the existence of a magnetically ordered state at low temperatures, although a number of different interpretations were put forward as to how they order. The group of Blinc et al. [10] suggested, on the basis of electron spin resonance (ESR), that the spin state was more like a spin glass than a normal ferromagnet, while Zakhidov et al. [11] suggested that the observed effects in ESR are due to super-paramagnetism, whereby large lumps of spins would order as a result of a magnetic field. At that time all experiments were done on fine powder samples, so it could be argued that the surface-to-volume ratio of these particles (up to $30 \%$ of all the buckyballs could be in the first two layers of each granule) could lead to some strange effects; or worse, since TDAE is sensitive to air, the surface could be degraded in some way. So it was very difficult at this point to decide what exactly is the intrinsic state. Nevertheless, it was established from low-field radio-frequency ESR [12] that in the absence of external field there was a small but finite internal field which was present only below $T_{c}$. The presence of such an internal field was confirmed later by muon spin resonance [13] and so the fact that there is a net ferromagnetic component to the spin order was established.

\section{New degrees of freedom associated} with molecular orientation effects

The molecular nature of these

ferromagnets gives rise to some rather unusual effects associated with the motion of $\mathrm{C}_{60}$ molecules. The rotational degrees of freedom of the fullerenes was soon found to have some important consequences for the low-temperature spin correlations. In fullerene solids, particularly containing $\mathrm{C}_{60}$, the buckyballs are so nearly spherical that they are able to rotate almost freely at room temperature. This rotation starts to slow down at lower temperatures - usually below $240 \mathrm{~K}$ - and eventually the molec- ules freeze into preferential positions, such that the maximum charge density (the $\mathrm{C}=\mathrm{C}$ double bond) on one ball faces the minimum (the centre of a hexagon or pentagon) on the neighbouring one. It turns out that a near-perfect threedimensional ordering which accommodates all the nearest neighbour buckyballs can be achieved in this way for $\mathrm{C}_{60}[14]$. This effect is also found in TDAE- $\mathrm{C}_{60}$ which leads to an effective exchange interaction between spins on the $\mathrm{C}_{60}$ molecules which is crucially dependent on their orientational order [15]. Since the molecular ordering can be influenced by quenching, for example, we find manifestly different macroscopic magnetic properties depending on how the sample is cooled [24].

A perhaps more suprising effect has been observed when the sample is slowly cooled in an external magnetic field. Seemingly the application of a magnetic field helps in the ordering of the molecules in a way that is favourable for the establishment of a spin-ordered state at low temperatures and $\mathrm{T}_{\mathrm{c}}$ increases by 8 $\mathrm{K}$ to $24 \mathrm{~K}$ by the application of a magnetic field above $T_{c}$ (no field need be applied in the low-temperature state). Although the detailed mechanism of how this occurs is not yet clear, since the local moments are starting to freeze in at about $150 \mathrm{~K}$ [25], we might expect that the coupling of the spins to the magnetic field can in principle influence the molecular orbiting by the spin-orbit interaction.

These orientational effects also have some uncomfortable experimental consequences. Precisely because of the above-mentioned peculiar dependence on sample history, experimenters working in the field often found that their results were somewhat different from sample to sample and day to day and group to group, which can of course create confusion when it comes to interpreting the results. As more is becoming known and understood about these materials, these problems are gradually being solved.

\section{The electronic ground state of fulle- rene magnets}

A first and foremost issue from the point of view of understading the ferromagnetic state in TDAE- $\mathrm{C}_{60}$ is whether we are dealing with itinerant or local moments. In the original report of the discovery of ferromagnetism in TDAE$\mathrm{C}_{60}$, it was thought that - because of the relatively high room temperature

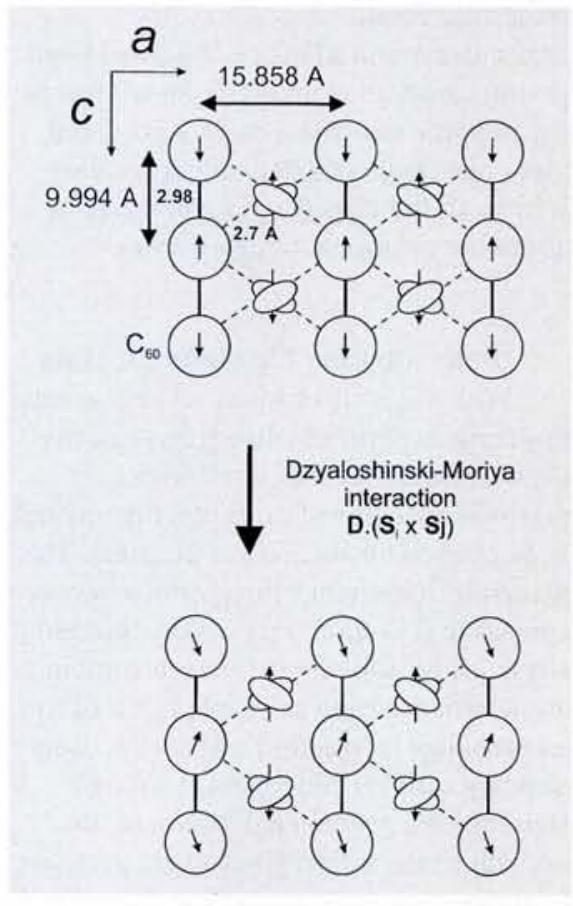

Fig. 3. The Dzyaloshinsky-Moriya (D-M) model for a spin-canted weak magnetic state in TDAE- $C_{60}$ assumes the strongest exchange interaction between $\mathrm{C}_{60}$ spins to be antiferromagnetic along the $c$-axis, but spin canting perpendicular to the c-axis is suggested to be responsible for the weak net ferromagnetic moment

conductivity $\left(10^{-2} \Omega^{-1} \mathrm{~cm}^{-1}\right)$ of the powder samples and a Pauli-like temperatureindependent susceptibility - TDAE- $\mathrm{C}_{60}$ is a metal. Since TDAE transfers one electron to $\mathrm{C}_{60}$ - a fact confirmed by a number of experiments [24] - the highest occupied molecular orbital (HOMO) $t_{14}$ level of $\mathrm{C}_{60}$, which is six-fold degenerate, is filled by one electron, and in principle a metallic ground state could be expected. However, subsequent measurements which were designed to determine if the ground state is indeed metallic, failed to confirm this. In particular, conductivity measurements first on pressed powder pellets [16], then on thin films [17] and finally on single crystals [20] all gave an activated temperature dependence more characteristic of a disordered semiconductor than a metal or semi-metal. The molecular ordering effects which were discussed in the previous section also become quite apparent in the electrical conductivity, modulating the inter-ball electronic hopping probabilities and giving rise to an activation energy $E_{\mathrm{a}}$ in the conductivity which is different in the low-temperature molecularly ordered state compared to high temperatures when the buckyballs are rotating [20]. Since the electron hopping rate is usually faster than the 
molecular rotation frequency, the molecular orientations can be considered pseudo-random from the point of view of the hopping electrons, and a disordered glass-like frequency dependent conductivity $[20]$ is obtained at temperatures above the rotational freezing point.

\section{Understanding the magnetic state}

With the issue of itinerant versus local moments experimentally settled and the crystal structure established with a reasonable degree of certainty, the way has been opened for theoretical progress. The principle of exchange interactions between spins is that Coulomb repulsion between electrons on adjacent orbitals in combination with the Pauli principle can lead to an exchange interaction which effectively strongly couples their spins. Although Heisenberg's original derivation of the exchange interaction gives a spin parallel ferromagnetic arrangement as the ground state, it is an anti-ferromagnetic (AFM) ground state that is nature's way. Fullerene charge-transfer salts are no exception and of the numerous charge-transfer compounds that have been synthesized, either a paramagnetic or antiferromagnetic state is the rule and ferromagnetism the exception. However, the occurence of AFM appears to be a prerequisite for an FM state at lower temperatures and so far all the materials which show a ferromagnetic transition also show a departure from the Curie law in the susceptibility characteristic of AFM at much higher temperatures.

Following this observation, combined with the fact that the saturation magnetization in most samples is only a fraction of what would be expected for a fully spin-ordered ferromagnet, it has been proposed that the net magnetization arises from a small canting of the $\mathrm{C}_{60}$ spins in $\mathrm{TDAE}^{+} \mathrm{C}_{60}^{-}$via a spin-orbital effect, the so-called Dzyaloshinsky-Morya (D-M) interaction (Fig. 3).

The predicted net magnetization in this model would point perpendicular to the direction of the strongest (AFM) exchange interaction direction which is in apparent agreement with susceptibility anisotropy measurements on single crystals [21]. This suggestion is very appealing theoretically, but nevertheless some pieces of the puzzle are still missing. The most important problem is that the magnitude of the D-M interaction should be proportional to the magnitude of the spin-orbit coupling, but this is known

\section{Molecular magnetism in other materials}

Ferromagnetism in molecular solids is a relatively new discovery, first reported by J.Miller and A.Epstein [1] in an organometallic salt containing dacamethylferrocenium tetracyanoethenide $\left[\mathrm{FeCp}_{2}\right]^{+}[\mathrm{TCNE}]^{-}$with a Curie temperature of $4.8 \mathrm{~K}$. Since the compound contains Fe with an unfilled shell of electrons, its spin contributes to the magnetization and therefore strictly speaking it is not an organic ferromagnet. In this material the cations and anions alternate along chains and are ferromagnetically coupled along the chain axis. However,

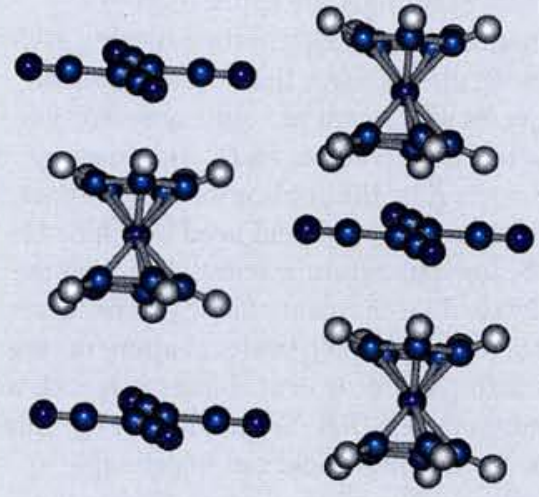

Fig. 1. The crystal structure of $\left(\mathrm{FeC} \mathrm{p}_{2}\right)+\mathrm{TCNE}^{-}$showing the alternating chains of the donor and acceptor molecules

it is the weak interactions between chains which are believed to be necessary to establish long range ferromagnetic order in this material (Fig. 1).

A more impressive compound of this type discovered by the same group is the vanadium solid complex with incorporated solvent molecules $\mathrm{V}$ (TCNE) ${ }_{2} 1 / 2 \mathrm{CH}_{2} \mathrm{Cl}_{2}$ which is a room temperature ferromagnet with $\mathrm{T}_{c}$ estimated at around $400 \mathrm{~K}$ (its $\mathrm{T}_{\mathrm{c}}$ can only be estimated since it decomposes at $350 \mathrm{~K})$. Although the structure of the material is relatively complex and has not been completely determined, it is believed that the TCNE binds to four different $\mathrm{V}$ atoms, each of which in

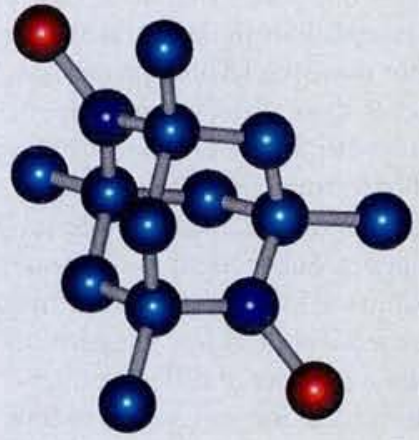

Fig. 2. The non-ionic organic ferromagnet TMDAD is composed of molecular units shown above

turn is surrounded by up to six nitrogens from the TCNE. Unfortunately it is also impressively reactive (explosive) upon contact with air or moisture and is not very useful for practical applications.

True organic magnetism means that the spins which order ferromagnetically are exclusively in p states such as in fullerene magnetism of TDAE- $\mathrm{C}_{60}$ or in the cobaltocene-doped fullerenes, where the the metal atom has a closed shell configuration and no spin. Apart from the fullerene compounds, ferromagnetic ordering has also been found in some other nitrogen compounds which are not donor-acceptor compounds. The best characterized is 4-nitrophenyl nitronyl nitroxide $\left(\mathrm{T}_{\mathrm{c}}=0.6 \mathrm{~K}\right)$, but the compound with the highest $\mathrm{T}_{\mathrm{c}}$ was discovered recently by the group of Andre Rassat, namely 1,3,5,7-tetramethyl-2,6-diaza-adamantine- $\mathrm{N}, \mathrm{N}^{\prime}$ dioxyl (TMDAD) with a Curie temperature of $1.48 \mathrm{~K}$ [3] shown in Fig. 2. More thorough reviews of the properties of these materials are given by Gatteschi [2] and Kahn [4].

[1] J.S. Miller and A.J. Epstein, J.Am.Chem.Soc. 109, 3850 (1987)

[2] D. Gatteschi, Adv. Mat. 6, 635 (1994)

[3] R. Chiarelli, M.A. Novak, A. Rassat and J.L. Tholence, Nature 363, 147 (1993)

[4] O. Kahn: Molecular Magnetism, (VCH Publishers Inc., New York, 1993) 
from the ESR g-shift to be very small, in apparent contradiction with the D-M theory. Moreover, the theory predicts a highly anisotropic susceptibility, in contradiction with most recent susceptibility data. On the other hand, the many spin-glass characteristics like divergence of the higher-order susceptibilities [18] and slow time-relaxation behaviour [12] indicates clearly that the spin ordering is frustrated and the FM state is only the net result of a number of competing interactions of these materials all conspiring to make our life interesting (or difficult, depending on your point of view).

\section{The future}

At the end of his 1928 paper on the magnetic exchange interactions, Heisenberg specified two conditions for the existence of ferromagnetism [2]:

1. The crystal lattice must be such that each atom has at least 8 nearest neighbours.

2. The principal quantum number of the electrons responsible for magnetism must be $>3$.

His ideas on the exchange interaction have of course become one of the corner stones of modern solid state theory, but his concluding remarks indicate how perilous predictions can be. Not only is the principal quantum number in organic materials less than 3 , but also the spin interactions responsible for the collective state come from very complex molecular orbitals with new degrees of freedom which have little to do with either of Heisenberg's maxims.

In spite of the fact that common wisdom supposes that the spin-spin interactions are weak in organic materials, there is no a priori reason to suppose that ferromagnetism in molecular organic materials should exist only at low temperatures. Indeed the anti-ferromagnetic state exists in many fullerene materials well above room temperature. Although besides TDAE- $\mathrm{C}_{60}$ only two other fullerene materials have been reproducibly synthesized showing low-temperature correlated spin ordering, namely $\mathrm{CoCp}_{2}-$ dispiromethano-fullerene[19] $(\mathrm{Tc}=8 \mathrm{~K})$ and very recently $\mathrm{CoCp}_{2}$-APMF (Fig. 4) with $\mathrm{T}_{\mathrm{c}}=20 \mathrm{~K}$, the latter shows features of a meta-magnetic state, where the spin ordering is reversibly induced by the application of an external field.

The importance of the discovery of the latter material is not so much the fact that it now holds the record $\mathrm{T}_{c}$, but that it

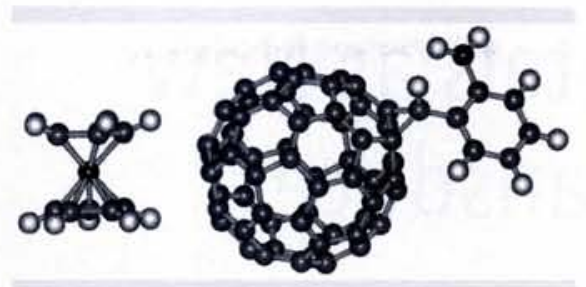

Fig. 4. The building blocks for $C O C \mathrm{p}_{2}$-APMF are cobaltocene and a fullerene nitrogen-containing derivative 3-aminophenyl-methano-[60]fullerene. This material currently has the record high $T_{\text {c for a }}$ molecular organic ferromagnet. Its structure is not yet known

shows that neither the spin $\left(\mathrm{CoCp}_{2}{ }^{+}\right.$has spin 0 , while $\mathrm{TDAE}^{+}$has spin $1 / 2$ ) nor shape of the electron donor is crucial for the existence of FM order. We can now infer with confidence that it is the fullerenes which are the building blocks of molecular magnetism in these materials, which opens up the search for new materials in a wider area of chemical synthesis.

There are very good reasons to try and find new organic ferromagnets with higher $\mathrm{T}_{c} s$ from the point of view of applications. Organic materials in general do not require high-temperature synthesis and are soluble in organic solvents, making processing easier for magnetic storage media for example. Moreover they are very light, which makes them potentially interesting for transformer cores for example, especially in space and for mobile applications. Yet the fundamental challenge remains in understanding a spin correlated state composed entirely of $p$ electrons, and it is clear that a good understanding of the origin of the molecular ferromagnetism in TDAE- $\mathrm{C}_{60}$ would be a significant aid in designing new magnetic materials with stronger ferromagnetic interactions.

As a final remark, it is rather amusing that it was actually a search for new forms of molecular carbon in interstellar space by H.W.Kroto, R.E.Smalley and R.F.Curl - for which they were awarded the 1996 Nobel prize for chemistry - that lead to the discovery and development of high-temperature organic ferromagnets, and now a new field in solid state physics. It just shows how difficult it is to predict future discoveries in science. Nevertheless, the race for achieving hightemperature organic molecular ferromagnetism is now definitely on.

\section{References}

[1] P.W. Anderson: Exchange in Insulators; superexchange, direct exchange and double exchange, in Magnetism vol.1, Eds. G.T. Rado and H. Suhl, (Academic press, New York 1963)

[2] W. Heisenberg, Z.Phys. 49, 619 (1928)

[3] P-M. Allemand, K.C. Khemani,

A. Koch, F. Wudl, K. Holczer, S. Donovan, G. Gruner, J.D. Thompson, Science 253, 301 (1991)

[4] W. Kraetschmer et al., Nature 347, 354 (1990)

[5] A.F. Hebard et al., Nature 350, 600 (1991)

[6] P.W. Stevens et al., Nature 355, 331 (1992); L. Golic et al.: Fullerenes and Fullerene

Nanostructures, Eds.

H. Kuzmany, J. Fink, M. Mehring, S. Roth, p. 351 (World Scientific, 1996)

[7] H. Klos, I. Rystau, W. Schutz,

B. Gotschy, A. Skiebe,A. Hirsch, Chem. Phys.Lett. 224, 333 (1994)

[8] Y. Li et al., Sol.Stat.Comm. 86, 475, (1993)

[9] A. Mrzel, A. Omerzu, D. Mihailovic, to be published

[10] P. Venturini et al., Int.J.Mod.Phys.B 6, 3947 (1993)

[11] K. Tanaka et al., Phys. Rev. B 47, 7554 (1992)

[12] P. Cevc et al., Sol.Stat. Comm. 90, 543 (1994)

[13] A. Lappas et al., Science 267, 1799 (1995)

[14] W.I.F. David et al., Europhys. Lett. 18, 219

(1992)

[15] K. Tanaka et al., Chem.Phys.Lett. 259, 574 (1996)

[16] A. Schilder et al., Phys. Rev.Lett. 73, 1299

(1994)

[17] A. Hassanien et al.: Physics and Chemistry of Fullerenes and Derivatives, Eds. H. Kuzmany, J.

Fink M. Mehring, S. Roth, p.489 (World Scientific 1995)

[18] K. Tanaka et al., Chem.Phys.Lett. 237, 123 (1995)

[19] P. Venturini et al.: Progress in Fullerene

Research, Eds. H. Kuzmany,

J. Fink M. Mehring, S. Roth, p.514 (World Scientific 1994)

[20] A. Omerzu, D. Mihailovic, S. Tomic,

O. Milat, N. Biskup, Phys.Rev.Lett. 77, 2045 (1996)

[21] R. Blinc, K. Pokhodnia, P. Cevc,

D. Arcon, A. Omerzu, D. Mihailovic, P. Venturini, L. Golic, Z. Trontelj, J. Luznik, Z. Jeglicic and J.

Pirnat, Phys. Rev.Lett. 76, 523 (1996)

[22] P.W. Stephens, D. Cox, J.W. Lauher,

L. Mihaly, J.B. Miley, P-M. Allemand,

A. Hirsch, K. Holczer, Q. Li, J.D. Thompson, F.

Wudl, Nature 355, 331 (1992)

[23] Dzyaloshinsky, J.Phys.Chem.Sol. 4, 241 (1958), T. Moriya, Phys.Rev.Lett. 4, 228 (1960) [24] D. Mihailovic, D. Arcon, P. Venturini, R. Blinc, A. Omerzu, P. Cevc, Science 268, 400 (1995)

[25] A. Mrzel, P. Cevc, A. Omerzu and

D. Mihailovic, Phys Rev B 53, R2922 (1996) 